\title{
NEPHROGENIC SYSTEMIC FIBROSIS: MINI-REVIEW
}

\author{
doi: $10.1590 / \mathrm{S} 1807-59322009000500017$
}

Juliano Sacramento Mundim, Sabrina de Castro Lorena, Rosilene Motta Elias, João Egídio Romão Júnior

\section{CASE REPORT}

The patient was a 43-year-old male who had been diagnosed with Alport syndrome and dialytic chronic kidney disease in 1998. In November 2006, he was admitted to the hospital due to septic shock secondary to a hemodialysis catheter infection that developed into a femoral prosthesis infection.

One month later, the patient underwent two MRI scans as preparation for the surgical prosthesis substitution. A gadolinium-based contrast agent was used with a gadodiamide dose of $0.15 \mathrm{mmol} / \mathrm{kg}$ in each exam, with a one-week interval between exams.

Two months after the MRI, the patient had thickening and hardening of the skin of the right forearm (Figure 1) that extended to the remaining limbs, as well as to a band lesion in the left lower limb (Figure 2). The remaining physical examination was normal.

The laboratory findings included an increase in C-reactive protein to $52 \mathrm{mg} / \mathrm{L}$, with a slight increase in serum ferritin to $320 \mathrm{ng} / \mathrm{mL}$. Other tests were normal, such as the hemosedimentation velocity and serum albumin concentration.

The pulmonary function test and the echocardiogram were both normal. A computed tomography (CT) scan of the affected areas showed diffuse thickening of the muscle, skin and subcutaneous tissue. The patient then underwent muscle and skin biopsies.

Nephrogenic systemic fibrosis (NSF) was confirmed in the skin and muscle biopsies. Immunohistochemical analysis showed the presence of CD34+, CD68+ and factor XIIIa+

Nephrology Department, Hospital das Clínicas da Faculdade de Medicina da Universidade de São Paulo - São Paulo/SP, Brazil.

Email: julianomundim@ig.com.br

Tel.: 55113069.6570

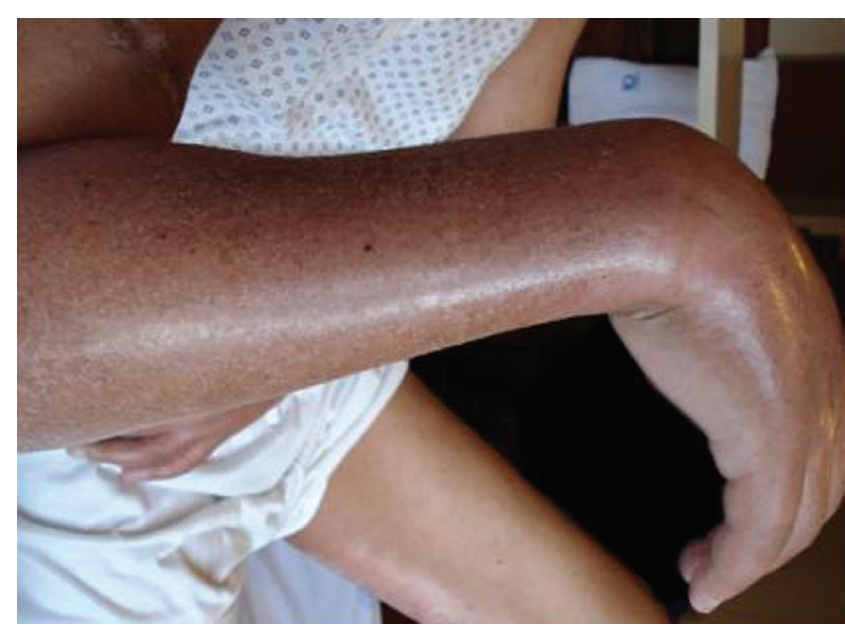

Figure 1 - Thickening and hardening of the skin of the right forearm

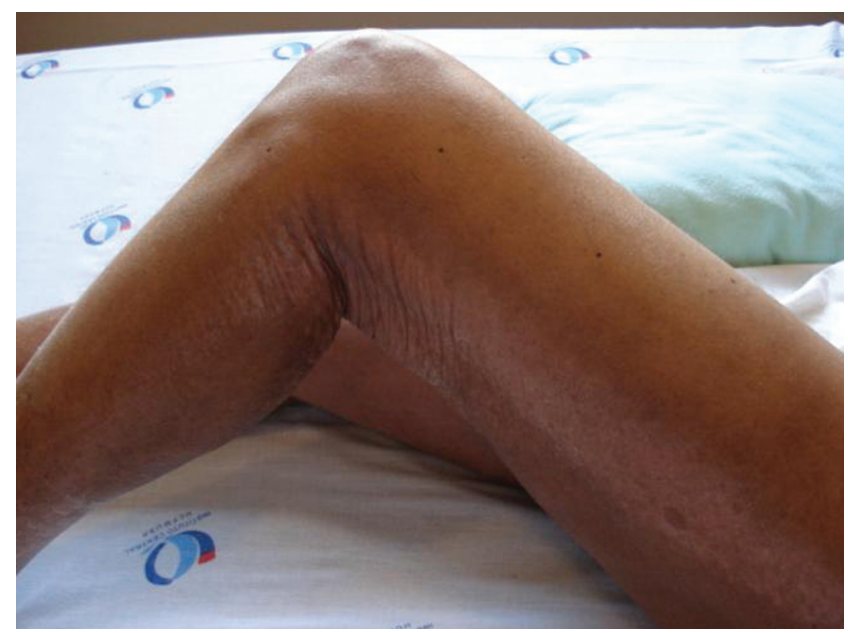

Figure 2 - Band lesion in the left lower limb

mono- and multinucleated cells, and electron microscopy showed gadolinium deposition in the muscle and skin.

Treatment with thalidomide and sodium thiosulphate was started, but no significant improvement has been observed. 


\section{DISCUSSION}

NSF is a devastating and irreversible disease that affects the skin and other tissues in patients with impaired kidney function (especially in patients with creatinine clearance < $30 \mathrm{ml} / \mathrm{min}$ ). ${ }^{1,2}$ This report describes the first case of NSF in Brazil to the best of our knowledge.

In NSF, the mean time between exposure to gadolinium and the onset of the lesions is 25 days, ranging from 2 to 75 days. ${ }^{1}$

The characteristic clinical findings are lesions of the extremities, especially the hands and feet, as well as the absence of head and trunk lesions. The affected areas can initially present local edema and develop hard and erythematous plaques, resulting in dermal and epidermal fibrosis, or even fibrosis of deeper tissues such as muscles and internal organs. ${ }^{2}$

There are no specific laboratory findings associated with NSF. To attain a definite diagnosis, it is necessary to perform a biopsy of the affected area, to observe evidence of fibrosis in the form of markers such as CD34 and CD68, and to note gadolinium deposits in the affected area. ${ }^{1-3}$

The differential diagnoses include dermatological pathologies that lead to skin thickening such as scleromyxedema, scleroderma, eosinophilic fasciitis and calciphylaxis. ${ }^{3}$

Several treatments for NSF have been tested, including both systemic treatments such as corticoids, sodium thiosulfate, cyclophosphamide and thalidomide, and topical treatments such as phototherapy. However, none of these agents have yet been shown to be definitively effective in reversing or even decreasing the progress of the disease. ${ }^{4}$

In individuals with normal kidney function, gadolinium rapidly distributes between the plasma and the interstitium. It has a half-life of 2 hours and is eliminated through glomerular filtration without any contribution from tubular secretion, with clearance varying from 1.1 to $1.6 \mathrm{ml} / \mathrm{kg} /$ $\min$. More than $95 \%$ of the injected dose is eliminated in 24 hours, and less than $3 \%$ is eliminated in the stool. ${ }^{5}$

In patients with advanced kidney failure (stage 5), the pharmacokinetics of the gadolinium complexes are altered. Due to their relatively low molecular weight (500 kDa), small volume of distribution $(0.28 \mathrm{l} / \mathrm{kg})$ and low protein binding, they are easily removed by hemodialysis, but not by peritoneal dialysis. ${ }^{5}$

A study showed that the half-life of gadolinium in a patient with chronic kidney disease was 34.3 hours. It decreased to 2.6 hours in patients undergoing hemodialysis, whereas in the patients undergoing peritoneal dialysis, it increased to 52.7 hours. ${ }^{6}$

The existing data in the literature allow for the following recommendations for preventing and minimizing the risks of acquiring this disease; however, there is no treatment for this pathology at the present date. ${ }^{4,7}$

The first and best measure is to suggest that all patients undergoing MRI should be evaluated for the presence of kidney disease, thus identifying those at increased risk of developing NSF, such as: patients with acute kidney disease, mainly associated with liver failure; patients that received kidney or liver transplants and have kidney disease; patients with kidney disease in stages 4 or 5 not undergoing dialytic therapy; ${ }^{5,10}$ and patients undergoing hemodialysis or peritoneal dialysis. Kidney function assessment is recommended for patients with elevated serum creatinine levels to ensure safety when performing the MRI. When a patient at risk of developing NSF is identified, the use of gadolinium should be avoided, unless such an examination is mandatory. ${ }^{5,8-10}$ If the MRI with gadolinium is truly essential, the lowest possible dose should be used, ${ }^{1,8-11}$ and multiple exposures to gadolinium should be avoided. The patient must be informed of the risks, benefits and alternatives. All at-risk patients that are exposed to examinations with contrast must undergo a clinical assessment. The use of gadolinium must be avoided in patients with acute kidney failure until the kidney function has recovered. ${ }^{8,9}$

Among patients with chronic kidney disease undergoing hemodialysis that are exposed to gadolinium, three hemodialysis sessions should be performed, with the first beginning within three hours after the exposure. It must be emphasized that the efficacy of hemodialysis in preventing NSF is unknown. ${ }^{8}$

For patients with chronic kidney disease undergoing peritoneal dialysis that are exposed to gadolinium, the number of dialysis bag changes should be increased, or the automatic peritoneal dialysis prescription should be extended to at least 48 hours. Also, hemodialysis should be considered for those that have vascular access and have been exposed to high or multiple doses of gadolinium, or if the nephrologist deems it necessary. ${ }^{8}$

Among patients with acute kidney disease or chronic kidney disease in stages 4 or 5 that are not undergoing dialysis and were exposed to gadolinium, it is not currently possible to estimate the risk of developing NSF. The decision to submit patients to dialysis must be assessed on an individual basis, by evaluating the risks and benefits in a joint decision among the nephrologist, the patient and the clinician. ${ }^{8}$

In conclusion, NSF is a progressive and potentially fatal disease that has been described in patients with acute or chronic kidney disease with a glomerular filtration rate $<30$ $\mathrm{mL} / \mathrm{min} / 1.73 \mathrm{~m}^{2}$ who are not undergoing dialysis therapy 
and are exposed to gadolinium-based contrast agents. To date, no therapy or combination of therapies has shown any consistent benefit for recovery of kidney function in NSF patients.

\section{REFERENCES}

1. Mendoza FA, Artlett CM, Sandorfi N, Latinis K, PieraVelazquez $\mathrm{S}$, Jimenez SA. Description of 12 cases of nephrogenic fibrosing dermopathy and review of the literature. Semin Arthritis Rheum. 2006 35:238-49.

2. Ting WW, Stone MS, Madison KC, Kurtz K. Nephrogenic fibrosing dermopathy with systemic involvement. Arch Dermatol. 2003;139:9036.

3. Cowper SE, Robin HS, Steinberg SM, Su LD, Gupta S, LeBoit PE. Scleromyxedemalike

cutaneous disease in renal-dialysis patients. Lancet. 2000;356:1000-1.

4. Linfert DR, Schell JO, Fine DM. Treatment of nephrogenic systemic fibrosis: limited options but hope for the future. Semin Dial. 2008;21:155-9.

5. Perazella MA, Rodby RA. Gadolinium use in patients with kidney disease: A cause for concern. Semin Dial. 2007;20:179-85.

6. Joffe P, Thomsen HS, Meusel M. Pharmacokinetics of gadodiamide injection in patients with severe renal insufficiency and patients undergoing hemodialysis or continuous ambulatory peritoneal dialysis. Acta Radiol. 1998;5:491-502.
7. Food and Drug Administration [Cyted 2007 july 12] Available from: URL: http://www.fda.gov/cder/drug/advisory/gadolinium

8. Perazella MA, Reilly RF. Nephrogenic systemic fibrosis: recommendations for gadolinium-based contrast use in patients with kidney disease. Semin Dial. 2008; 21:171-3.

9. Perazella MA. How should nephrologists approach gadolinium-based contrast imaging in patients with kidney disease? Clin J Am Soc Nephrol. 2008;3:649-51.

10. Deo A, Fogel M, Cowper SE. Nephrogenic systemic fibrosis: A population study examining the relationship of disease development to gadolinium exposure. Clin J Am Soc Nephrol. 2007;2:264-7.

11. Moschella SL, Kay J, Mackool BT, Liu V. Case records of the Massachusetts General Hospital. Weekly clinicopathological exercises. Case 35004. A 68 year old man with end stage renal disease and thickening of the skin. N Engl J Med. 2004;351:2219-27. 